\title{
Uma análise de conteúdo de crenças relacionadas com a AIDS entre participantes em O.N.G.s.
}

\author{
Marco Antonio de Castro Figueiredo \\ Luciana Nogueira Fioroni \\ Universidade de São Paulo
}

\begin{abstract}
Uma amostra de sete sujeitos, pertencentes a Organizações Não Governamentais (ONGs) de Ribeirão Preto, foi entrevistada através de procedimentos semi estruturados para verificação de cognições associadas a quatro categorias de conteúdos relacionados à AIDS: a doença em si, o tratamento, o paciente e prevenção. Uma análise de conteúdo das entrevistas foi processada sobre avaliações feitas por 10 juízes universitários, com base em procedimentos de cálculo das entropias relativas $(\mathrm{Hr})$, tomando-se, como critério, escores iguais ou inferiores ao percentil 33 das distribuições de $\mathrm{Hr}$ e verificando-se a fidedignidade dos julgamentos para cada categoria $(Z k>3.39 ; \mathrm{p}<.05)$. Foram identificadas crenças de forte conotação teleológica $(\mathrm{Hr}<.29)$ relacionadas com a morte, a vergonha dentro da família e o silêncio das pessoas para a categoria doença. A respeito do paciente, apareceram representações ligadas ao estigma, perda da cidadania e responsabilidade pela própria condição $(\mathrm{Hr}<.35)$. Confirmando dados de literatura, a visão do tratamento e da prevenção se respalda em crenças altamente socializadas $(\mathrm{Hr}<.39)$ que envolvem a participação da família e a atribuição de fortes estigmas por parte dos especialistas que atendem ao paciente.

Palavras-chave: Síndrome da Imunodeficiência Adquirida, Organizações Não Governamentais, Saúde Coletiva, Cognições.
\end{abstract}




\section{Introdução}

ação da comunidade organizada, na área de saúde,
vem se constituindo como forma alternativa para
suprir a demanda reprimida, não atendida pelos órgãos oficiais, principalmente com relação à abordagem da AIDS. Buscando superar uma série de dificuldades de ordem política, econômica e, principalmente técnica, tais esforços tentam complementar o atendimento hospitalar feito pelas equipes especializadas (Projeto Rocinha, 1992), proporcionando uma fonte importante de recursos informais para a prevenção, educação, suporte material e emocional (Longo, 1993; Clausson, 1989), além da luta pela cidadania da pessoa com AIDS. No entanto, tais iniciativas da sociedade esbarram nos limites de uma prática assistemática e sem outros recursos humanos além dos originados na própria atuação.

Por outro lado, o universo acadêmico se organiza de forma diversa, contaminado pela preocupação com a eficiência, produtividade e sistematização (Camargo, 1992; Freitas, 1993). Ao nível do atendimento técnico, apesar dos avanços, o suporte ao paciente HIV continua insuficiente quanto ao trato psicossocial do sujeito (Figueiredo \& Souza, 1993), tendo como característica a compartimentalização do fenômeno, com divisão de responsabilidades reduzidas a um campo específico de ação, implicando na perda da compreensão global dos problemas decorrentes da AIDS. Formados dentro de uma perspectiva técnica que desvitaliza a relação terapêutica, os profissionais de saúde transformam o paciente num quadro clínico a ser resolvido (Camargo, 1994).

Tais particularidades destes dois pólos da prática justificam buscar uma ação conjunta entre ciência e comunidade como forma mais adequada para o tratamento da AIDS enquanto fenômeno psicossocial. A aproximação destes dois elementos, enquanto objeto de estudo, visa a implementação de projetos de intervenção, através da participação efetiva de profissionais e agentes de comunidade, tanto ao nível da concepção como da atuação prática, complementando o nível de competência dos elementos envolvidos. Desta forma, as equipes 
multidisciplinares que tratam da questão da AIDS poderiam se constituir enquanto grupos de decisão, compostos por elementos de vários segmentos da sociedade, onde a real dimensão do problema e a melhoria do atendimento passariam pela inserção nos processos de organização do trabalho enquanto todo.

Tomando como referencial o componente cognitivo do Modelo Bifatorial de Atitudes proposto por Fishbein e Ajzen (Figueiredo, 1994; Fishbein \& Ajzen, 1975) o presente trabalho procura enfocar, na concepção de saúde/doença da comunidade, as formas com que os fenômenos decorrentes da AIDS e seu enfrentamento são representados, proporcionando informações subsidiárias para a identificação de potenciais participantes em projetos visando o atendimento psicossocial do paciente/portador do HIV.

Concebidas como complexos derivados de crenças e valores ligados a um fenômeno, determinando propensões comportamentais coerentes com estas cognições valorizadas, as atitudes são consideradas, dentro das abordagens afetivo-cognitivas, fontes importantes de informação sobre o comportamento e as formas sob as quais toda a atividade humana se manifesta. No presente trabalho estaremos nos atendo ao componente cognitivo da atitude, que possibilita verificar crenças e representações a respeito da AIDS dentro da comunidade, enquanto manifestação de seus elementos ligados às Organizações Não Governamentais. A opção por tomar como referência os agentes da comunidade que militam em Organizações Não Governamentais se fundamenta no caráter normativo destas, enquanto formadoras de opinião e por concentrar em seus quadros elementos ativos e de liderança na luta contra a AIDS.

O emprego deste referencial teórico se justifica pelo fato de viabilizar a evidência dos principais atributos relacionados à AIDS enquanto fenômeno, que poderiam determinar atitudes características do grupo estudado, respondendo ao objetivo principal da pesquisa, a identificação de elementos formadores da representação social da AIDS no âmbito da comunidade organizada. 


\section{Método}

\section{Sujeitos}

Foram entrevistados sete voluntários das duas Organizações Não Governamentais (ONGs) que atuam diretamente em programas de atendimento e prevenção à AIDS, na cidade de Ribeirão Preto, o Grupo de Apoio e Prevenção à AIDS (GAPA) e Brigada dos Militares Espiritas.

\section{Procedimento}

Foram realizadas entrevistas semi-estruturadas com o objetivo de evocar, enunciar e verificar crenças relacionadas com alguns aspectos da AIDS para, posteriormente, proceder uma análise de conteúdo de representações sociais dos sujeitos entrevistados, com referência ao fenômeno em questão. A entrevista foi realizada em três etapas, cujo procedimento é descrito no quadro 1.

Quadro 1. Fases da entrevista realizada e respectivos procedimentos.

\begin{tabular}{|l|l|}
\hline \multicolumn{1}{|c|}{ FASES } & \multicolumn{1}{c|}{ PROCEDIMENTOS } \\
\hline 1. Evocação & $\begin{array}{l}\text { É pedido ao sujeito que pense durante um minuto } \\
\text { em todas as coisas que julga importantes a } \\
\text { respeito do atributo estudado. }\end{array}$ \\
\hline 2. Enunciação & $\begin{array}{l}\text { Pede-se ao entrevistado que diga quais as três } \\
\text { coisas mais importantes que pensou, e o } \\
\text { entrevistador anota as respostas em uma folha. }\end{array}$ \\
\hline 3. Averiguação & $\begin{array}{l}\text { Pede-se ao entrevistado que complemente as } \\
\text { respostas dadas e o entrevistador esclarece } \\
\text { possiveis dúvidas remanescentes. }\end{array}$ \\
\hline
\end{tabular}

As verbalizações dos sujeitos foram transcritas sob forma de locuções e classificadas segundo quatro categorias, Doença, Tratamento, Paciente e Prevenção, cujos conteúdos são apresentados pelo quadro 2 .

As locuções obtidas foram analisadas e classificadas por dois juízes de nível universitário, visando a eliminação de conteúdos comuns. Os itens redundantes foram sintetizados em uma única locução para compor uma lista intermediária, objeto da análise de conteúdo. A fidedignidade dos julgamen- 
tos foi verificada com base no cálculo do coeficiente kappa (k) (Landis \& Koch, 1975).

Quadro 2 - Relação das categorias estudadas e respectivas subcategorias

\begin{tabular}{|c|l|}
\hline CATEGORIAS & \multicolumn{1}{|c|}{ SUBCATEGORIAS } \\
\hline \multirow{5}{*}{ DOENÇA } & 1. Origem \\
& 2. Contágio \\
& 3. Resposta social \\
& 4. Prognóstico \\
& 5. Conhecimento \\
& 6. Prevenção \\
& 7. Diagnóstico \\
& 8. Razão necessária \\
\hline \multirow{5}{*}{ TRATAMENTO } & 1. Eficácia \\
& 2. Política de saúde \\
& 3. Omissão de socorro \\
& 4. Atendimento comunitário \\
& 5. Necessidades do profissional \\
& 6. Necessidades do paciente \\
& 7. Dificuldades técnicas \\
& 8. Recursos materiais \\
\hline & 1. Conscientização \\
& 2. Educação \\
& 3. Necessidade \\
& 4. Informação \\
& 5. Preconceito \\
& 6. Condições sócio-econômicas \\
\hline
\end{tabular}

A análise de conteúdo destas locuções foi realizada com base em protocolos específicos submetidos a dez juízes de nível universitário, cuja tarefa consistiu na classificação das locuções nas subcategorias pré-determinadas, apresentadas anteriormente.

A seleção de conteúdos foi feita com base no cálculo da Entropia Relativa (Hr) das locuções estudadas, procedimento que vem sendo utilizado com bastante freqüência na análise de itens (Figueiredo \& Galera, 1981) e no levantamento de crenças salientes modais para elaboração de questionário de atitudes (Figueiredo, 1986; Figueiredo \& Palma, 1992).

O conceito "entropia" faz parte do corpo teórico da Teoria da Informação (Edwards, 1967) e pode ser utilizado para estudos descritivos, uma vez que desempenha um papel análogo ao 
da variância ou ao da correlação da estatística clássica (Stephaneck, 1975). No nosso caso, a distribuição dos julgamentos, para cada item, foi avaliada no sentido de selecionar os conteúdos cuja quantidade média de informação garantisse sua inclusão numa dada categoria. A entropia dos itens foi calculada a partir da seguinte equação:

$$
H=\sum_{i=1}^{n} p_{i} \log _{2} p_{i}
$$

Onde: $p_{i}$ é a probabilidade da inserção nas várias categorias.

Uma vez que o número de categorias presentes nos julgamentos variou, de item para item, as entropias obtidas foram transformadas em valores relativos, segundo a seguinte equação:

$$
H_{\text {rel }}=\frac{H_{i}}{H_{\max }}
$$

Onde: $\mathrm{Hi}$ é a entropia do item estudado

Hmax é a entropia máxima do sistema

Assim, tomando como critério o percentil 33 da distribuição dos scores de Hr, foram selecionadas as locuções de conteúdos representativos para as quatro categorias estudadas.

\section{Resultados e discussão}

Considerando os estudos de fidedignidade dos julgamentos relativos à análise inicial para eliminação de redundâncias, a tabela 1 apresenta os resultados do coeficiente $k$ para as quatro categorias estudadas.

Tabela 1. Resultados do coeficiente k para os estudos de fidedignidade de julgamentos, estudos iniciais $(p<.05)$.

\begin{tabular}{|c|c|c|c|c|}
\hline & DC & TR & PC & PV \\
\hline$k$ & $+.42^{*}$ & $+22^{*}$ & $+.48^{*}$ & $+.65^{*}$ \\
Zk & 6.88 & 3.39 & 8.81 & 4.75 \\
\hline
\end{tabular}


Com relação aos estudos de Entropia para a análise de conteúdo, os valores críticos dos pontos de corte (percentil 33) para seleção de itens variaram entre .29 e .35 , permitindo identificar os conteúdos típicos para cada categoria, apresentados na tabela 2:

Tabela 2. Conteúdos selecionados para as categorias estudadas e respectivas $\mathrm{Hr}$

\begin{tabular}{|c|c|c|c|}
\hline CATEGORIA & ITEM & $\begin{array}{l}\text { CONTEÚDOS MODAIS } \\
\text { (freqüências) }\end{array}$ & $\mathrm{Hr}$ \\
\hline \multirow{5}{*}{$\begin{array}{c}\text { A } \\
\text { DOENÇA }\end{array}$} & $\overline{01}$ & Origem (07) & $.26^{*}$ \\
\hline & 02 & Razão necessária (09) & $.14^{*}$ \\
\hline & 04 & Razão necessária (10) & $\mathrm{Z}^{*}$ \\
\hline & 09 & Resposta social (06) & $.29^{*}$ \\
\hline & 10 & Resposta social (09) & $.14^{*}$ \\
\hline \multirow{4}{*}{$\begin{array}{c}\text { B } \\
\text { TRATAMENTO }\end{array}$} & 11 & Eficácia (06) & $.39^{*}$ \\
\hline & 12 & Política de saúde & $.39^{*}$ \\
\hline & 13 & Omissão de socorro (09) & $.14^{*}$ \\
\hline & 16 & Necessidade do profissional (10) & $Z^{*}$ \\
\hline \multirow{10}{*}{$\begin{array}{c}\text { C } \\
\text { PACIENTE }\end{array}$} & 19 & Prevenção (10) & $\mathrm{Z}^{*}$ \\
\hline & 21 & Prevenção (08) & $.22^{*}$ \\
\hline & 23 & Estigmatização (09) & $.14^{*}$ \\
\hline & 24 & Estigmatização (10) & $\mathrm{Z}^{*}$ \\
\hline & 26 & Estigmatização (07) & $.35^{*}$ \\
\hline & 29 & Necessidades (10) & $\mathrm{Z}^{*}$ \\
\hline & 30 & Efeito psicológico (07) & $.35^{*}$ \\
\hline & 31 & Efeito psicológico (06) & $.39^{\star}$ \\
\hline & 32 & Estigmatização (07) & $.26^{*}$ \\
\hline & 34 & Estigmatização (09) & $.14^{*}$ \\
\hline \multirow{4}{*}{$\begin{array}{c}\text { D } \\
\text { PREVENÇÃO }\end{array}$} & 36 & Educação (07) & $.35^{*}$ \\
\hline & 37 & Necessidade (07) & $.35^{\star}$ \\
\hline & 39 & Preconceito (09) & $.14^{*}$ \\
\hline & 40 & Condições sócio-econômicas (08) & $.28^{*}$ \\
\hline
\end{tabular}

Com base nestes resultados, uma síntese dos conteúdos obtidos foi realizada. O quadro 3 apresenta os conteúdos selecionados para cada categoria de análise e respectivas subcategorias: 
Quadro 3. Síntese da Análise de Conteúdo realizada para as quatro categorias $(n=23)$.

\begin{tabular}{|c|c|c|}
\hline CATEGORIAS & $\begin{array}{c}\text { SUB- } \\
\text { CATEGORIAS }\end{array}$ & CONTEÚDOS \\
\hline \multirow{3}{*}{$\begin{array}{c}\text { A } \\
\text { DOENÇA }\end{array}$} & Origem & 01. A AIDS é uma doença antiga. \\
\hline & $\begin{array}{l}\text { Razão } \\
\text { necessária }\end{array}$ & $\begin{array}{l}\text { 02. A AIDS veio por causa de maus hábi- } \\
\text { tos. } \\
04 \text {. A AIDS leva as pessoas a pensarem } \\
\text { na morte. }\end{array}$ \\
\hline & $\begin{array}{l}\text { Resposta } \\
\text { social }\end{array}$ & $\begin{array}{l}\text { 09. A AIDS causa vergonha na família do } \\
\text { paciente. } \\
\text { 10. O medo de contrair o HIV leva as } \\
\text { pessoas a não conversarem sobre o } \\
\text { assunto. }\end{array}$ \\
\hline \multirow{4}{*}{$\begin{array}{l}\text { B } \\
\text { TRATAMEN- } \\
\text { TO }\end{array}$} & Eficácia & $\begin{array}{l}\text { 11. Atualmente a evolução da doença } \\
\text { leva à morte do paciente. }\end{array}$ \\
\hline & $\begin{array}{l}\text { Política de } \\
\text { saúde }\end{array}$ & $\begin{array}{l}\text { 12. Os laboratórios de saúde não se } \\
\text { interessam em curar a doença. }\end{array}$ \\
\hline & $\begin{array}{l}\text { Omissão de } \\
\text { socorro }\end{array}$ & $\begin{array}{l}13.0 \text { s hospitais e serviços odontológicos } \\
\text { se recusam a atender pessoas infectadas } \\
\text { pelo HIV. }\end{array}$ \\
\hline & $\begin{array}{l}\text { Necessidade } \\
\text { do profissional }\end{array}$ & $\begin{array}{l}\text { 16. O profissional que atende o paciente } \\
\text { com AIDS necessita de suporte psicoló- } \\
\text { gico para continuar a fazê-lo. }\end{array}$ \\
\hline \multirow{4}{*}{$\begin{array}{l}\text { C } \\
\text { PACIENTE }\end{array}$} & Prevenção & $\begin{array}{l}\text { 19. O grupo de homossexuais procura } \\
\text { controlar a transmissão da doença. } \\
\text { 21. Os heterossexuais não controlam a } \\
\text { transmissão da doença. }\end{array}$ \\
\hline & Estigmatização & $\begin{array}{l}\text { 23. Os pacientes gays e drogaditos não } \\
\text { são aceitos quando voltam para casa. } \\
\text { 24. A pessoa com AIDS perde a cidada- } \\
\text { nia. } \\
\text { 26. O paciente HIV é responsabilizado } \\
\text { pela própria condição. } \\
\text { 32. Os pacientes seriam menos discrimi- } \\
\text { nados se as pessoas tivessem mais } \\
\text { interesse a respeito da doença. } \\
\text { 34. Os pacientes adultos sofrem mais do } \\
\text { que as crianças. }\end{array}$ \\
\hline & $\begin{array}{l}\text { Efeito } \\
\text { Psicológico }\end{array}$ & $\begin{array}{l}\text { 30. O paciente com AIDS revolta-se e } \\
\text { quer contaminar outras pessoas. } \\
31.0 \text { conhecimento do diagnóstico causa } \\
\text { efeitos irreversíveis na vida do paciente. }\end{array}$ \\
\hline & Necessidades & $\begin{array}{l}\text { 29. O paciente HIV precisa de um suporte } \\
\text { social e afetivo. }\end{array}$ \\
\hline \multirow{4}{*}{$\begin{array}{l}\text { D } \\
\text { PREVENÇÃO }\end{array}$} & Educação & $\begin{array}{l}\text { 29. O paciente HIV precisa de um suporte } \\
\text { social e afetivo. }\end{array}$ \\
\hline & Necessidades & $\begin{array}{l}\text { 37. O uso de preservativos nas relações } \\
\text { sexuais é um meio importante de salvar } \\
\text { vidas. }\end{array}$ \\
\hline & Preconceito & $\begin{array}{l}\text { 39. Há preconceito contra o uso de cami- } \\
\text { sinha entre os alunos de medicina. }\end{array}$ \\
\hline & $\begin{array}{l}\text { Condições } \\
\text { sócio- } \\
\text { econômicas }\end{array}$ & $\begin{array}{l}\text { 40. As pessoas mais pobres têm maiores } \\
\text { dificuldades de se prevenirem devido à } \\
\text { falta de informação. }\end{array}$ \\
\hline
\end{tabular}


A análise de conteúdo realizada permitiu verificar para cada categoria os conteúdos modais que, de certa forma, representam as cognições associadas aos aspectos ligados à AIDS e que podem ser considerados como significantes para uma análise posterior de propensões e atitudes de pessoas envolvidas com atividades de ONGs no que se refere à AIDS.

a. Considerando conteúdos relacionados à doença em si, prevaleceram os pensamentos de caráter social envolvendo o pensamento na morte, a vergonha dentro da família e o silêncio das pessoas, levadas pelo medo de contrair o HIV. Além disso, respostas teleológicas relacionadas aos costumes apareceram associadas ao surgimento da doença devido à hábitos censurados pela sociedade.

b. Da mesma forma, para o paciente, apareceram conteúdos de forte conotação social envolvendo a estigmatização, $o$ efeito psicológico e as necessidades da pessoa infectada. Para as pessoas que trabalham nas ONGs a perda da cidadania, a responsabilidade pela própria doença e a rejeição pela família determinam a estigmatização do paciente. Em contrapartida, o efeito psicológico no paciente é visto como consequiência irreversível, deixando-o propenso a contaminar outras pessoas.

Nota-se por estes conteúdos preocupações com a família e com respostas afetivas que determinam tanto o isolamento do paciente quanto o silêncio das pessoas não infectadas, identificando o fenômeno que Daniel (1989) chama de morte social, colocando o paciente frente a constrangimentos determinados pela recusa ao tratamento (Calabrese, Kelley, Cullen \& Locker, 1991; Carlisle, 1992) e pela exclusão nos mais elementares direitos à cidadania (Biancarelli, 1992; Castanheira \& Vassalo, 1992; "Curso da USP", 1989; Greig, 1987).

c. No que se refere ao tratamento, foram selecionados conteúdos ligados à omissão de socorro e política de saúde, tais como a falta de interesse por parte dos laboratórios em curar a doença. O tratamento médico não é visto como eficaz, já que a evolução do quadro clínico leva à morte da pessoa contaminada, onde os efeitos das condições de atendimento incidem também sobre o profissional, este necessita de suporte para continuar atendendo. 
Esta visão do tratamento é confirmada pela literatura, que relata estigmas arraigados por parte dos especialistas (Gallop, 1991; Gillon, 1987; Hardie, 1992; Kelly, 1987), coincidente com os relatos da produção do conhecimento científico, denotando uma aproximação da forma mergulhada da percepção da comunidade, enquanto usuária do serviço de saúde.

Muitos trabalhos têm relatado dificuldades psicológicas de profissionais de saúde com relação às formas de interação (Calabrese et al, 1991; Gallop, 1991), envolvendo sentimentos como antipatia (Kelly, 1987) desconforto (Gillon, 1987) e perplexidade (Carlisle, 1992) frente às condições físicas e emocionais em que o paciente se encontra. $\mathrm{O}$ tratamento do paciente com AIDS deixa seqüelas que podem se tornar irreversíveis na vida do profissional. Presa de exigências conflitantes, que envolvem simultaneamente responsabilidade e auto-preservação (Figueiredo, 1994), o profissional se vê frente a situações ambivalentes que necessitam ser esclarecidas. Os resultados obtidos no presente trabalho denotam uma clara percepção destas dificuldades por parte dos sujeitos entrevistados.

d. Finalmente, a visão de prevenção se respalda em crenças que envolvem a participação da família e o uso de preservativos, muito comuns nos programas de disseminação de informação para a população (Coverdale \& Aruffo, 1992; Vasconcelos, 1992; Williams, 1991). Além disso, um componente de forte conotação sócio-econômica implica em crenças sobre maiores dificuldades de prevenção no interior das camadas mais pobres da população, motivado pela falta de informação. Curiosamente parece existir, dentro dos grupos não governamentais estudados, crenças de que alunos de medicina têm preconceito quanto ao uso da camisinha. Tal aspecto parece denotar uma suspeita de que o preconceito já esteja estabelecido durante a formação do profissional de saúde, indicando alguns limites psicossociais, lançando dúvidas com respeito à preparação do profissional para o suprimento das necessidades do paciente enquanto indivíduo.

De um modo geral pode-se dizer que as crenças levantadas no presente estudo levam a indicadores interessantes para 
avaliação afetivo-cognitiva das propensões de participantes de ONGs quanto às atividades de tratamento e prevenção da AIDS na comunidade. Como era de se esperar, conteúdos de forte conotação social foram encontrados, envolvendo principalmente os limites determinados pelo estigma e pelas dificuldades psicossociais do atendimento à AIDS. Por outro lado, sinalizam na direção do núcleo familiar como uma importante fonte de recursos para prevenção e atendimento do portador e paciente com AIDS.

Todas estas questões suscitadas pelos sujeitos estudados nos remetem às bases do fenômeno AIDS, colocando a descoberto algumas contradições que determinam as dificuldades mais importantes para o tratamento psicossocial do paciente, independente do lugar onde é atendido: se nos hospitais e serviços de saúde, ou se em domicílio, ou pela comunidade organizada. Ainda que a sociedade organizada possa ser a resposta "legítima" ao fenômeno social, dada sua condição de "elemento nele mergulhado" (Figueiredo, 1994, p.130), nem sempre constitui a forma adequada; por outro lado, por mais que as equipes técnicas estejam instrumentalizadas técnica, teórica e metodologicamente, sua compreensão do fenômeno restringe-se ao seu corpo especializado e a ação se ressente, na prática. Assim, se a resposta da comunidade não se ajusta à ação sistemática, ou seja, à forma disciplinadamente científica, as tentativas da técnica esbarram nos limites da especialidade da ação, tornada parcelar.

Futuros projetos deverão tratar de forma mais detalhada os conteúdos aqui levantados, onde um possível rearranjo de tais conteúdos em dimensões de avaliação poderão servir de base para a elaboração de um instrumento de diagnóstico para melhor aproveitamento dos recursos humanos que a comunidade tem à disposição na luta contra a AIDS.

Abstract: A content analysis of beliefs related to AIDS among N. G. O.s participants

A sample of seven subjects belonging to Non-Government

Organizations (NGOs) of Ribeirão Preto was interviewed 
using semi-structured procedures to determine the cognitions associated with four content categories related to AIDS: the disease in itself, treatment, patient, and prevention. Content Analysis of the interviews was processed using evaluations made by 10 university judges on the basis of procedures of relative entropy $(\mathrm{Hr})$ calculations, using as criterion scores equal to or less than the 33rd percentile of the $\mathrm{Hr}$ distributions and determining the reliability of judgment for each category $(\mathrm{Zk}>3.39 ; \mathrm{p}<.05)$. Beliefs with a strong teleological connotation were identified $(\mathrm{Hr}<.29)$ related to death, shame within the family and people silence for the disease category. With respect to the patient, representations linked to the stigma, loss of citizenship and responsibility for his own condition were detected $(\mathrm{Hr}<.35)$. Confirming literature data, the vision of treatment and prevention is based on highly socialized beliefs $(\mathrm{Hr}<.39)$ involving participation of the family and the attribution of strong stigmas on the part of the specialists providing care to the patient.

Key words: Acquired Immunodeficiency Syndrome, NonGovernmental Organizations, Public Health, Cognitions.

\section{Referências}

Biancarelli, A. (1992, 07 de maio). Sindicato dos proprietários de escola decide manter a proibição, apesar de críticas de representantes da Saúde. Folha de São Paulo, p.4, cad.3.

Calabrese, L. H., Kelley, D. M., Cullen, R. J., \& Locker, G. (1991). Physicians' attitudes, beliefs and practices regarding AIDS Health Care Promotion. Arch. Intern. Med., 151(6), 1157-60.

Camargo Jr., K. R. (1994). AIDS e a AIDS das ciências. História, Ciências, Saúde-Manguinhos., 1(1), 35-60.

Camargo Jr., K. R. (1992). (Ir)racionalidade Médica: os Paradoxos da Clínica. Physis, Revista de Saúde Coletiva., 2(1), 203-28.

Carlisle, D. (1992). Right to refusal. Nurs. Times. 88(7), 18-9.

Castanheira, I., \& Vassalo, C. (1992). A reação do mundo dos negócios ao assédio a AIDS. Exame, 24(7), 60-6.

Clausson, N. I. (1989). Le SIDA: la résponse communitaire. Rev. Can. Santé Pub., 80, 20-5. 
Coverdale, J. H., \& Aruffo, J. F. (1992). AIDS and family planning counselling of psychiatrically ill women in Community Mental Health Clinics. Com. Mental Health J., 28(1), 13-9.

Curso da USP proibe pesquisa com aidéticos. (1989, 20 de agosto). Folha de São Paulo, Caderno D, p.8.

Daniel, H. (1989). Vida antes da morte/Life before death. Rio de Janeiro: Tipografia Jabuti.

Edwards, W. (1967). The theory of decision making. In: W. Edwards \& A. Twersky (Orgs.), Decision Making. Harmondsworth: Penguin Books.

Figueiredo, M. A. C. (1994). Profissionais de saúde e SIDA. Um estudo diferencial com base no modelo afetivo-cognitivo de Fishbein e Ajzen (1975). Tese de livre docência, não-publicada, Universidade de São Paulo, Ribeirão Preto.

Figueiredo, M. A. C., Souza, L. B. (1993). Atitudes frente à AIDS: análise da adequação de um modelo afetivo/cognitivo para estudos diferenciais com profissionais de saúde. Medicina (Ribeirão Preto), 26(3), 393407.

Figueiredo, M. A. C. (1986). Algumas tentativas de caracterização de aspectos afetivos, cognitivos e comportamentais das Emoções, através da aplicação do modelo teórico de Fishbein e Ajzen sobre Atitudes. Tese (doutorado). Instituto de Psicologia da Universidade de São Paulo, São Paulo.

Figueiredo, M. A. C., \& Palma, C. M. S. (1992). Uma análise de conteúdo sobre crenças de pais de pessoas com AIDS a respeito da doença do paciente e do tratamento que é dispensado. Resumos da XXII Reunião Anual da Sociedade Brasileira de Psicologia, Ribeirão Preto.

Figueiredo, M.A.C., \& Galera, C.A. (1981). Construção e validação de um questionário de atitudes, através do cálculo de Entropias dos itens utilizados. Resumos da XI Reunião Anual da Sociedade de Psicologia de Ribeirão Preto, Ribeirão Preto.

Fishbein, M., \& Ajzen, I. (1975). Belief, Attitude, Intention and Behavior: an introduction to theory and research. Massachussets: AddisonWesley.

Freitas, D. M. V. (Coord.). (1993). Reforma curricular de Graduação em Enfermagem. Escola de Enfermagem de Ribeirão Preto, Universidade de São Paulo. Rev. Lat. Am. Enf., 1(2), 35-52.

Gallop, R. M. (1991). The Knowledge, Attitudes and Concerns of Hospital Staff about AIDS. Can. J. Pub. Health., 82(6), 409-12.

Gillon, R. (1987). Refusal to treat AIDS and HIV positive patients. British. Med. J., 294(6583), 1332-3.

Greig, D. N. H. (1987). Refusal to treat AIDS and HIV positive patients (Letter) British. Med. J., 295(6590), 120-1.

Hardie, J. (1992). Addressing the fears of HIV transmission in dental practice. J. Can. Dent. Assoc., 58(3), 193-6. 
Kelly, J. A. (1987). Stigmatization of AIDS patients by physicians. Am. J. Public Health, 77(7), 789-91.

Landis, J.R., \& Koch, G.G. (1975). A review of statistical methods in the analysis of data arising from observer reliability studies (parts 1 and 2). Statistica Neerlandica, 29, 101-23 and 151-61.

Longo, P. H. (1993). Programa Pegação. Prevenção para rapazes que se prostituem no Rio de Janeiro. AIDS \& DST. Experiências que funcionam., 1(2), 8-9.

Projeto Rocinha (1992). A comunidade assume a própria saúde. AIDS \& DST. Experiências que funcionam., 1(1), 4-5.

Stephaneck, P. (1975). Psicologia Industrial I. (Apostila) Departamento de Psicologia e Educação, FFCLRP USP, Ribeirão Preto.

Vasconcelos, A. (Coord.) (1992). Projeto Casa de Passagem. Projetos de intervenção comportamental em execução. AIDS \& DST. Experiências que funcionam, $1,10$.

Williams, B. A. (1991). Improving Community Support for HIV and AIDS prevention through national partnerships. Pub. Health Rep., 106(6), 672-7.

Marco Antonio de Castro Figueiredo é Professor Associado junto ao Departamento de Psicologia e Educação da FFCLRP USP. Doutor em Psicologia Experimental pela Universidade de São Paulo. Endereço para correspondência: FFCLRP-USP, Departamento de Psicologia e Educação, Avenida dos Bandeirantes 3900, Monte Alegre, CEP 14040-901, Ribeirão Preto (SP). Luciana Nogueira Fioroni é bolsista de Iniciação Científica do Conselho de Desenvolvimento Científico e Tecnológico, CNPq. 